\title{
AARP
}

\section{State Taxes and Revenue Options in West Virginia: Survey Methodology and Annotated Questionnaire}

February 2022

\section{Methodology}

In December 2021, AARP engaged MBE Research (MBE) to conduct a quantitative research study among registered voters in West Virginia. MBE completed a total of 700 live telephone interviews including 35\% conducted among a cell phone sample. Both landline and cell phone sampling were used for this research, with the sample drawn randomly from a list of West Virginia voters age 18 and older purchased from Aristotle.

The base sample of 700 respondents yields a maximum statistical error of $\pm 3.63 \%$ at the $95 \%$ level of confidence. Some percentages of some questions may exceed $100 \%$ due to rounding or the use of multiple response question formats. All data have been weighted by age and gender according to 2021 West Virginia state voter database statistics

\section{Screening Questions}

S1. Our study is interested in the opinions of certain age groups. Could you please tell me your age as of your last birthday?

\begin{tabular}{|l|c|}
\hline & Total (\%) \\
\hline Unweighted $n$ & 700 \\
\hline $18-34$ & 13 \\
\hline $35-49$ & 35 \\
\hline $50-64$ & 18 \\
\hline $65+$ & 34 \\
\hline
\end{tabular}

S2. And just to confirm, are you a resident of West Virginia?

\begin{tabular}{|l|c|}
\hline Unweighted $n$ & 700 \\
\hline Yes [CONTINUE] & 100 \\
\hline No [TERMINATE] & - \\
\hline Don't know [TERMINATE] & - \\
\hline Refused [TERMINATE] & - \\
\hline
\end{tabular}




\section{AARP}

S3. What is your 5 -digit zip code?

S4. And are you registered to vote in West Virginia?

\begin{tabular}{|l|c|}
\hline & Total (\%) \\
\hline Unweighted $n$ & 700 \\
\hline Yes [CONTINUE] & 100 \\
\hline No [TERMINATE] & - \\
\hline Don't know [TERMINATE] & - \\
\hline Refused [TERMINATE] & - \\
\hline
\end{tabular}

S5. How often would you say you vote in state and local elections for Governor, Congressional representatives, and Mayors? Would you say you: always vote in state and local elections, nearly always vote, sometimes vote, seldom vote, or have never voted in a state or local election?

\begin{tabular}{|l|c|}
\hline & Total (\%) \\
\hline Unweighted $n$ & 700 \\
\hline Always & 73 \\
\hline Nearly always & 14 \\
\hline Sometimes & 9 \\
\hline Seldom & 4 \\
\hline Never & - \\
\hline Would rather not say [VOL] & - \\
\hline Not sure [VOL] & - \\
\hline Refused [VOL] & - \\
\hline
\end{tabular}

\section{Main}

1. How would you rate the current condition of the economy in West Virginia? Is it excellent, good, fair, or poor?

\begin{tabular}{|l|c|}
\hline & Total (\%) \\
\hline Unweighted $n$ & 700 \\
\hline Excellent & 1 \\
\hline Good & 12 \\
\hline Fair & 38 \\
\hline Poor & 48 \\
\hline Not sure & 2 \\
\hline
\end{tabular}




\section{AARP}

2. And how would you rate your own personal financial situation these days......would you say you are in excellent shape, good shape, fair shape, or in poor shape financially? [REVERSE RESPONSE SCALE EVERY OTHER TIME]

\begin{tabular}{|l|c|}
\hline & Total (\%) \\
\hline Unweighted $n$ & 700 \\
\hline Excellent & 9 \\
\hline Good & 36 \\
\hline Fair & 37 \\
\hline Poor & 17 \\
\hline Not sure & - \\
\hline Refused & 1 \\
\hline
\end{tabular}

3. There has been a lot of talk in the news recently about people considering leaving West Virginia to live elsewhere. In the past year, have you personally considered leaving West Virginia?

\begin{tabular}{|l|c|}
\hline & Total (\%) \\
\hline Unweighted $n$ & 700 \\
\hline Yes & 28 \\
\hline No & 72 \\
\hline Not sure & - \\
\hline Refused & - \\
\hline
\end{tabular}

4. [IF Q3 $=$ Yes] Which of the following best describes your reason for moving from West Virginia and residing in another state? Is it for... [INSERT AND RANDOMIZE a-I; ANCHOR k; MULTIPLE RESPONSES ALLOWED]

\begin{tabular}{|l|c|}
\hline & Total (\%) \\
\hline Unweighted $n$ & 195 \\
\hline Better job opportunities & 43 \\
\hline Better weather or climate & 29 \\
\hline Lower taxes & 26 \\
\hline Better kindergarten to 12th grade school system & 11 \\
\hline Higher education opportunities & 18 \\
\hline Health or health care reasons & 20 \\
\hline A safer community or lower crime rate & 18 \\
\hline Family reasons - be with or live closer to other family & 21 \\
\hline members & \\
\hline Better run state or local government & 23 \\
\hline Lower overall cost of living & 17 \\
\hline Other reason (specify): & 17 \\
\hline Not sure & 3 \\
\hline Refused & - \\
\hline
\end{tabular}




\section{AARP}

5. And in the past year, have you heard someone you know talk about leaving West Virginia?

\begin{tabular}{|l|c|}
\hline & Total (\%) \\
\hline Unweighted $n$ & 700 \\
\hline Yes & 51 \\
\hline No & 49 \\
\hline Not sure & - \\
\hline Refused & - \\
\hline
\end{tabular}

6. [IF Q5 $=$ Yes] And what is their reason for wanting to leave West Virginia to live somewhere else? Is it...?

\begin{tabular}{lc|} 
& Total (\%) \\
\hline Unweighted $n$ & 353 \\
\hline Better job opportunities & 70 \\
\hline Better weather or climate & 13 \\
\hline Lower taxes & 12 \\
\hline Better kindergarten to 12th grade school system & 11 \\
\hline Higher education opportunities & 15 \\
\hline Health or health care reasons & 6 \\
\hline A safer community or lower crime rate & 7 \\
\hline Family reasons - be with or live closer to other family & 12 \\
\hline members & 11 \\
\hline Better run state or local government & 8 \\
\hline Lower overall cost of living & 15 \\
\hline Other reason (specify): & 4 \\
\hline Not sure & - \\
\hline Refused &
\end{tabular}

7. Now, I'm going to read a list of issues facing many West Virginians today. For each one, please tell me if you think that issue should be a high priority $(H)$, medium priority $(M)$, or low priority $(L)$ for the state to address over the next few years.

a. Public education $\mathrm{k}-12$

\begin{tabular}{|l|c|}
\hline & Total (\%) \\
\hline Unweighted $n$ & 700 \\
\hline High priority & 75 \\
\hline Medium priority & 20 \\
\hline Low priority & 5 \\
\hline Don't know & 1 \\
\hline
\end{tabular}


b. Taxes

\begin{tabular}{|l|c|}
\hline & Total (\%) \\
\hline Unweighted $n$ & 700 \\
\hline High priority & 43 \\
\hline Medium priority & 41 \\
\hline Low priority & 14 \\
\hline Don't know & 3 \\
\hline
\end{tabular}

c. Health care

\begin{tabular}{|l|c|}
\hline & Total (\%) \\
\hline Unweighted $n$ & 700 \\
\hline High priority & 69 \\
\hline Medium priority & 26 \\
\hline Low priority & 4 \\
\hline Don't know & 1 \\
\hline
\end{tabular}

d. Housing

\begin{tabular}{|l|c|}
\hline & Total (\%) \\
\hline Unweighted $n$ & 700 \\
\hline High priority & 40 \\
\hline Medium priority & 42 \\
\hline Low priority & 16 \\
\hline Don't know & 2 \\
\hline
\end{tabular}

e. Prescription drugs

Unweighted $n$

Total (\%)

High priority

Medium priority

700

Low priority

62

Don't know

28

7

2

f. Air and water quality

\begin{tabular}{|l|c|}
\hline & Total (\%) \\
\hline Unweighted $n$ & 700 \\
\hline High priority & 45 \\
\hline Medium priority & 35 \\
\hline Low priority & 20 \\
\hline Don't know & - \\
\hline
\end{tabular}


g. Higher education

\begin{tabular}{|l|c|}
\hline & Total (\%) \\
\hline Unweighted $n$ & 700 \\
\hline High priority & 47 \\
\hline Medium priority & 38 \\
\hline Low priority & 14 \\
\hline Don't know & 1 \\
\hline
\end{tabular}

h. Transportation and infrastructure

\begin{tabular}{|l|c|}
\hline & Total (\%) \\
\hline Unweighted $n$ & 700 \\
\hline High priority & 66 \\
\hline Medium priority & 27 \\
\hline Low priority & 6 \\
\hline Don't know & - \\
\hline
\end{tabular}

i. Utility costs

\begin{tabular}{|l|c|}
\hline & Total (\%) \\
\hline Unweighted $n$ & 700 \\
\hline High priority & 52 \\
\hline Medium priority & 39 \\
\hline Low priority & 7 \\
\hline Don't know & 4 \\
\hline
\end{tabular}

j. Legalization of recreational marijuana

\begin{tabular}{|l|c|}
\hline & Total (\%) \\
\hline Unweighted $n$ & 700 \\
\hline High priority & 27 \\
\hline Medium priority & 22 \\
\hline Low priority & 49 \\
\hline Don't know & 2 \\
\hline
\end{tabular}

k. Programs and services for older individuals, people with disabilities, and low-income individuals and families

\begin{tabular}{|l|c|}
\hline & Total (\%) \\
\hline Unweighted $n$ & 700 \\
\hline High priority & 58 \\
\hline Medium priority & 32 \\
\hline Low priority & 8 \\
\hline Don't know & 2 \\
\hline
\end{tabular}




\section{AARP}

I. Public safety

\begin{tabular}{|l|c|}
\hline & Total (\%) \\
\hline Unweighted $n$ & 700 \\
\hline High priority & 44 \\
\hline Medium priority & 41 \\
\hline Low priority & 15 \\
\hline Don't know & - \\
\hline
\end{tabular}

m. Jobs and the economy

\begin{tabular}{|l|c|}
\hline & Total (\%) \\
\hline Unweighted $n$ & 700 \\
\hline High priority & 78 \\
\hline Medium priority & 18 \\
\hline Low priority & 3 \\
\hline Don't know & 1 \\
\hline
\end{tabular}

n. Drug abuse and addiction

\begin{tabular}{|l|c|}
\hline & Total (\%) \\
\hline Unweighted $n$ & 700 \\
\hline High priority & 76 \\
\hline Medium priority & 15 \\
\hline Low priority & 7 \\
\hline Don't know & 1 \\
\hline
\end{tabular}

8. The state of West Virginia provides many services and programs for its residents. Please tell me if it is important or not important to you that West Virginia continue to maintain the following state services and programs. Let's begin.... would you say that it is important or not important to you that West Virginia maintain [ITEM]? [IF 'IMPORTANT, READ: and is that very or somewhat? [IF 'NOT IMPORTANT, READ: and is that not too important or not at all important?]

a. State and local parks and recreation

\begin{tabular}{|l|c|}
\hline & Total (\%) \\
\hline Unweighted $n$ & 700 \\
\hline Very important & 35 \\
\hline Somewhat important & 49 \\
\hline Not too important & 9 \\
\hline Not at all important & 5 \\
\hline Don't know & - \\
\hline
\end{tabular}


b. Home and community-based services for older West Virginians or people with disabilities (for example, meals on wheels, personal care assistance, transportation services, etc.)

Unweighted $n$

Very important

Somewhat important

Not too important

Not at all important

Don't know

\section{Total (\%)}

700

71

25

c. State and local infrastructure such as bridges and road maintenance and repair

\begin{tabular}{|l|c|}
\hline & Total (\%) \\
\hline Unweighted $n$ & 700 \\
\hline Very important & 71 \\
\hline Somewhat important & 27 \\
\hline Not too important & 2 \\
\hline Not at all important & - \\
\hline Don't know & - \\
\hline
\end{tabular}

d. Health services for children and low-income families

\begin{tabular}{|l|c|}
\hline & Total (\%) \\
\hline Unweighted $n$ & 700 \\
\hline Very important & 73 \\
\hline Somewhat important & 21 \\
\hline Not too important & 4 \\
\hline Not at all important & 2 \\
\hline Don't know & 1 \\
\hline
\end{tabular}

e. West Virginia public universities and colleges

\begin{tabular}{|l|c|}
\hline Unweighted $n$ & 700 \\
\hline Very important & 45 \\
\hline Somewhat important & 39 \\
\hline Not too important & 10 \\
\hline Not at all important & 6 \\
\hline Don't know & - \\
\hline
\end{tabular}


f. Public safety - fire, police, and other first responders

\begin{tabular}{|l|c|}
\hline & Total (\%) \\
\hline Unweighted $n$ & 700 \\
\hline Very important & 73 \\
\hline Somewhat important & 23 \\
\hline Not too important & 2 \\
\hline Not at all important & 1 \\
\hline Don't know & - \\
\hline
\end{tabular}

g. K-12 public education

\begin{tabular}{|l|c|}
\hline & Total (\%) \\
\hline Unweighted $n$ & 700 \\
\hline Very important & 76 \\
\hline Somewhat important & 17 \\
\hline Not too important & 6 \\
\hline Not at all important & 1 \\
\hline Don't know & - \\
\hline
\end{tabular}

9. Thinking about all taxpayers in West Virginia, please tell me if you think each of the groups (pay more than their fair share, less than their fair share, or about their fair share) in state income tax?

a. Low income families or individuals

Unweighted $n$

More than fair share

Less than fair share

About fair share

Don't know
Total (\%)

700

28

22

50

b. Middle income families or individuals

\begin{tabular}{|l|c|}
\hline & Total (\%) \\
\hline Unweighted $n$ & 700 \\
\hline More than fair share & 46 \\
\hline Less than fair share & 4 \\
\hline About fair share & 50 \\
\hline Don't know & - \\
\hline
\end{tabular}

c. High income families or individuals

\begin{tabular}{|l|c|} 
& Total (\%) \\
\hline Unweighted $n$ & 700 \\
\hline More than fair share & 15 \\
\hline Less than fair share & 51 \\
\hline About fair share & 34 \\
\hline Don't know & - \\
\hline
\end{tabular}


d. You and your family

\begin{tabular}{|l|c|}
\hline & Total (\%) \\
\hline Unweighted $n$ & 700 \\
\hline More than fair share & 41 \\
\hline Less than fair share & 4 \\
\hline About fair share & 56 \\
\hline Don't know & - \\
\hline
\end{tabular}

e. Local small businesses

\begin{tabular}{|l|c|}
\hline & Total (\%) \\
\hline Unweighted $n$ & 700 \\
\hline More than fair share & 41 \\
\hline Less than fair share & 9 \\
\hline About fair share & 50 \\
\hline Don't know & - \\
\hline
\end{tabular}

\section{f. Large corporations}

\begin{tabular}{|l|c|}
\hline & Total (\%) \\
\hline Unweighted $n$ & 700 \\
\hline More than fair share & 11 \\
\hline Less than fair share & 66 \\
\hline About fair share & 22 \\
\hline Don't know & - \\
\hline
\end{tabular}

10. In the last 12 months, or since last December, about how much have you heard, read, or seen about West Virginia's current tax system or any tax issues in the state - would you say a lot, some, not that much, or nothing at all?

\begin{tabular}{|l|c|}
\hline & Total (\%) \\
\hline Unweighted $n$ & 700 \\
\hline A lot & 16 \\
\hline Some & 32 \\
\hline Not much & 38 \\
\hline Nothing at all & 13 \\
\hline Not sure & 1 \\
\hline Refused & - \\
\hline
\end{tabular}




\section{AARP}

11. And in the past 12 months, or since last December, how much have you heard, read or seen about the reduction or elimination of the state income tax in West Virginia?

\begin{tabular}{|l|c|}
\hline & Total (\%) \\
\hline Unweighted $n$ & 700 \\
\hline A lot & 12 \\
\hline Some & 29 \\
\hline Not much & 24 \\
\hline Nothing at all & 35 \\
\hline Not sure & - \\
\hline Refused & - \\
\hline
\end{tabular}

12. The state is considering a proposal to gradually eliminate the state income tax for all West Virginians. While this could mean money back into the pockets of taxpayers, it could also reduce state revenue that is used to support critical state services and programs like health care, infrastructure, education, and public safety. Thinking about this, which of the following statements best represents your view? Do you think West Virginia should.....

\begin{tabular}{|c|c|}
\hline & Total (\%) \\
\hline Unweighted $n$ & 700 \\
\hline $\begin{array}{l}\text { Eliminate the state income tax and either [raise other state } \\
\text { taxes] and/or [reduce spending on state programs and } \\
\text { services] } \\
\text { [REVERSE ORDER "raise other state taxes" AND "reduce } \\
\text { spending on state programs and services" EVERY } \\
\text { OTHER RESPONDENT] }\end{array}$ & 14 \\
\hline $\begin{array}{l}\text { Keep the state income tax but change the income tax } \\
\text { rates so that lower and middle-income people pay less, } \\
\text { and higher-income people pay more, OR }\end{array}$ & 59 \\
\hline $\begin{array}{l}\text { Keep the state income tax as it is - don't increase taxes or } \\
\text { reduce spending on state programs and services }\end{array}$ & 27 \\
\hline
\end{tabular}

13. [ASK IF Q12=a] You stated a preference for eliminating the state income tax and either [raising other state taxes] and/or [reducing spending on state programs and services] [SHOW SAME ORDER AS DISPLAYED IN PREVIOUS QUESTION]

Which statement best represents your view?

\begin{tabular}{|l|c|}
\hline & Total (\%) \\
\hline Unweighted $n$ & 95 \\
\hline $\begin{array}{l}\text { Raise other state taxes - don't reduce spending on state } \\
\text { programs and services }\end{array}$ & 19 \\
\hline $\begin{array}{l}\text { Reduce spending on state programs and services - don't } \\
\text { raise other state taxes }\end{array}$ & 38 \\
\hline $\begin{array}{l}\text { Do both - raise other state taxes and reduce spending on } \\
\text { state programs and services }\end{array}$ & 44 \\
\hline
\end{tabular}




\section{AARP}

14. [IF Q13=b REDUCE SPENDING, READ: Even though you stated a preference for reducing spending rather than raising taxes] If West Virginia decided to eliminate the state income tax and raise other state taxes, please tell me if you would support or oppose each of the following options to fill the gap in revenue created by an elimination of the state's income tax....let's begin: Would you support or oppose...

a. Increasing the severance tax on coal and natural gas

Unweighted $n$

Strongly support

Somewhat support

Somewhat oppose

Strongly oppose
Total (\%)

700

16

28

13

44

b. Increasing the state sales tax

\begin{tabular}{|l|c|} 
& Total (\%) \\
\hline Unweighted $n$ & 700 \\
\hline Strongly support & 7 \\
\hline Somewhat support & 29 \\
\hline Somewhat oppose & 19 \\
\hline Strongly oppose & 44 \\
\hline
\end{tabular}

c. Adopting a sales tax on professional services such as legal and accounting services

\begin{tabular}{|l|c|}
\hline & Total (\%) \\
\hline Unweighted $n$ & 700 \\
\hline Strongly support & 5 \\
\hline Somewhat support & 26 \\
\hline Somewhat oppose & 30 \\
\hline Strongly oppose & 37 \\
\hline
\end{tabular}

d. Increasing the state gasoline tax

\begin{tabular}{|l|c|}
\hline & Total (\%) \\
\hline Unweighted $n$ & 700 \\
\hline Strongly support & 7 \\
\hline Somewhat support & 5 \\
\hline Somewhat oppose & 30 \\
\hline Strongly oppose & 58 \\
\hline
\end{tabular}

e. Increasing property taxes

\begin{tabular}{|l|c|}
\hline & Total (\%) \\
\hline Unweighted $n$ & 700 \\
\hline Strongly support & 3 \\
\hline Somewhat support & 3 \\
\hline Somewhat oppose & 6 \\
\hline Strongly oppose & 92 \\
\hline
\end{tabular}


f. Taxing recreational marijuana use if legalized

\begin{tabular}{|l|c|}
\hline & Total (\%) \\
\hline Unweighted $n$ & 700 \\
\hline Strongly support & 65 \\
\hline Somewhat support & 10 \\
\hline Somewhat oppose & 15 \\
\hline Strongly oppose & 8 \\
\hline
\end{tabular}

g. Adopting an estate tax

\begin{tabular}{|l|c|}
\hline & Total (\%) \\
\hline Unweighted $n$ & 700 \\
\hline Strongly support & 3 \\
\hline Somewhat support & 16 \\
\hline Somewhat oppose & 12 \\
\hline Strongly oppose & 64 \\
\hline
\end{tabular}

h. Increasing corporate income taxes

\begin{tabular}{|l|c|}
\hline & Total (\%) \\
\hline Unweighted $n$ & 700 \\
\hline Strongly support & 14 \\
\hline Somewhat support & 39 \\
\hline Somewhat oppose & 25 \\
\hline Strongly oppose & 21 \\
\hline
\end{tabular}

i. Adopting a luxury tax...for example on goods such as luxury boats, private jets, exotic sportscars, etc.

\begin{tabular}{|l|c|}
\hline & Total (\%) \\
\hline Unweighted $n$ & 700 \\
\hline Strongly support & 54 \\
\hline Somewhat support & 19 \\
\hline Somewhat oppose & 6 \\
\hline Strongly oppose & 21 \\
\hline
\end{tabular}




\section{AARP}

15. [IF Q13a, RAISE TAXES, READ: Even though you stated a preference for raising taxes rather than reducing spending] If West Virginia decided to eliminate the state income tax and reduce spending on state programs and services, please tell me if you would support or oppose each of the following options to fill the gap in revenue created by an elimination of the state's income tax....let's begin. Would you support or oppose the state reducing spending on...

a. State and local parks and recreation

\begin{tabular}{|l|c|}
\hline Unweighted $n$ & 18 \\
\hline Strongly support & 7 \\
\hline Somewhat support & 23 \\
\hline Somewhat oppose & 78 \\
\hline Strongly oppose & \\
\hline
\end{tabular}

b. Home and community-based services for older West Virginians or people with disabilities (for example, meals-on-wheels, personal care assistance, transportation services, etc.)

\begin{tabular}{|l|c|}
\hline & Total (\%) \\
\hline Unweighted $n$ & 18 \\
\hline Strongly support & 16 \\
\hline Somewhat support & 6 \\
\hline Somewhat oppose & 23 \\
\hline Strongly oppose & 65 \\
\hline
\end{tabular}

c. State and local infrastructure such as bridges and road maintenance and repair

\begin{tabular}{|l|c|}
\hline & Total (\%) \\
\hline Unweighted $n$ & 18 \\
\hline Strongly support & 9 \\
\hline Somewhat support & 16 \\
\hline Somewhat oppose & 10 \\
\hline Strongly oppose & 65 \\
\hline
\end{tabular}

d. Health services for children and low-income families

\begin{tabular}{lc|} 
& Total (\%) \\
\hline Unweighted $n$ & 18 \\
Strongly support & 55 \\
\hline Somewhat support & 15 \\
Somewhat oppose & 16 \\
\hline Strongly oppose & 14
\end{tabular}


e. West Virginia public universities and colleges

\begin{tabular}{|l|c|}
\hline & Total (\%) \\
\hline Unweighted $n$ & 18 \\
\hline Strongly support & 21 \\
\hline Somewhat support & 9 \\
\hline Somewhat oppose & 55 \\
\hline Strongly oppose & 16 \\
\hline
\end{tabular}

f. Public safety - fire, police, and other first responders

\begin{tabular}{|l|c|}
\hline & Total (\%) \\
\hline Unweighted $n$ & 18 \\
\hline Strongly support & 20 \\
\hline Somewhat support & 4 \\
\hline Somewhat oppose & 16 \\
\hline Strongly oppose & 60 \\
\hline
\end{tabular}

g. K-12 public education

\begin{tabular}{|l|c|}
\hline & Total (\%) \\
\hline Unweighted $n$ & 18 \\
\hline Strongly support & 16 \\
\hline Somewhat support & 51 \\
\hline Somewhat oppose & 20 \\
\hline Strongly oppose & 13 \\
\hline
\end{tabular}

\section{Demographics}

D1. A family caregiver is someone who provides UNPAID care for an older parent, spouse or other adult loved one who is ill, frail, elderly, or has a physical, mental, or emotional disability. This UNPAID care may include assisting with personal needs like bathing and dressing, household chores, meals, shopping, transportation, or managing finances, paperwork, or medical care.

a. Are you now or have you ever been a family caregiver - which means you provided UNPAID care to an adult loved one?

\begin{tabular}{|l|c|}
\hline & Total (\%) \\
\hline Unweighted $n$ & 700 \\
\hline Yes & 50 \\
\hline No & 50 \\
\hline Not sure & - \\
\hline Refused & - \\
\hline
\end{tabular}




\section{AARP}

b. [If yes to D1a] As a family caregiver, have you ever incurred any of the following expenses for the following services to help care for your loved one?

\begin{tabular}{|l|c|}
\hline Percentage Saying Yes & Total (\%) \\
\hline Unweighted $n$ & 397 \\
\hline Making modifications to your or your loved one's home & 66 \\
\hline $\begin{array}{l}\text { Purchasing equipment like a wheelchair, hearing aids, } \\
\text { vision aids, or wearable devices. }\end{array}$ & 66 \\
\hline Hiring a home health aide or personal care attendant & 36 \\
\hline $\begin{array}{l}\text { Using respite care to get a break from your caregiving } \\
\text { responsibilities }\end{array}$ & 26 \\
\hline Using adult day care & 12 \\
\hline Transporting your loved one to where they need to go & 89 \\
\hline
\end{tabular}

D2. Which of the following best describes your current employment status? Are you...?

\begin{tabular}{|l|c|}
\hline & Total (\%) \\
\hline Unweighted $n$ & 700 \\
\hline Self-employed full-time & 6 \\
\hline Self-employed part-time & 4 \\
\hline Employed full-time & 33 \\
\hline Employed part-time & 6 \\
\hline Retired and not working at all & 35 \\
\hline Unemployed and looking for work & 3 \\
\hline Or are you not in the labor force for other reasons & 10 \\
\hline Not sure [DO NOT READ] & 1 \\
\hline Refused [DO NOT READ] & 1 \\
\hline
\end{tabular}

D3. What is the highest level of education that you completed?

\begin{tabular}{|l|c|}
\hline & Total (\%) \\
\hline Unweighted $n$ & 700 \\
\hline $0-12^{\text {th }}$ grade (no diploma) & 8 \\
\hline High school graduate or equivalent & 31 \\
\hline Post-high school education (no degree) & 9 \\
\hline 2-year college degree & 16 \\
\hline 4-year college degree & 12 \\
\hline Post-graduate study (no degree) & 6 \\
\hline Graduate or professional degree & 16 \\
\hline Not sure [DO NOT READ] & 2 \\
\hline Refused [DO NOT READ] & 2 \\
\hline
\end{tabular}




\section{AARP}

D4. What is your current marital status?

\begin{tabular}{|l|c|}
\hline Unweighted $n$ & 700 \\
\hline Married & 68 \\
\hline Not married but living with your partner & 2 \\
\hline Separated & - \\
\hline Divorced & 7 \\
\hline Widowed & 7 \\
\hline Single and never married & 14 \\
\hline Not sure [DO NOT READ] & - \\
\hline Refused [DO NOT READ] & 1 \\
\hline
\end{tabular}

D5. [ASK ONLY IF MARRIED OR LIVING WITH PARTNER] Are you or your spouse or partner currently a member of A-A-R-P?

\begin{tabular}{|l|c|}
\hline & Total (\%) \\
\hline Unweighted $n$ & 490 \\
\hline Yes & 27 \\
\hline No & 71 \\
\hline Not sure [DO NOT READ] & 1 \\
\hline Refused [DO NOT READ] & - \\
\hline
\end{tabular}

D6. Are you of Hispanic, Spanish, or Latino origin or descent?

\begin{tabular}{|l|c|}
\hline & Total (\%) \\
\hline Unweighted $n$ & 700 \\
\hline Yes & 1 \\
\hline No & 97 \\
\hline Not sure [DO NOT READ] & - \\
\hline Refused [DO NOT READ] & 2 \\
\hline
\end{tabular}

D7. What is your race? Are you...

Unweighted $n$

White or Caucasian

Black or African American

American Indian or Alaska Native

Asian

Native Hawaiian or other Pacific Islander

Something else [DO NOT READ]

Not sure [DO NOT READ]

Refused [DO NOT READ]
Total (\%)

700

92

2

1

$-$

$-$

2

3 
D8. Do you consider yourself to be a ...?

\begin{tabular}{|l|c|} 
& Total (\%) \\
\hline Unweighted $n$ & 700 \\
\hline Democrat & 37 \\
\hline Republican & 38 \\
\hline Independent & 25 \\
\hline Something else? [RECORD ANSWER] & - \\
\hline Not sure [DO NOT READ] & - \\
\hline Refused [DO NOT READ] & - \\
\hline
\end{tabular}

D9. How would you characterize your political views?

\begin{tabular}{|l|c|}
\hline & Total (\%) \\
\hline Unweighted $n$ & 700 \\
\hline Very conservative & 21 \\
\hline Somewhat conservative & 28 \\
\hline Moderate & 24 \\
\hline Somewhat liberal & 16 \\
\hline Very liberal & 6 \\
\hline None of the above/something else [DO NOT READ] & 1 \\
\hline Not sure [DO NOT READ] & 3 \\
\hline Refused [DO NOT READ] & 2 \\
\hline
\end{tabular}

D10. We realize income is a private matter and so rather than ask you anything specific about your income, l'd like to ask you to please stop me when I get to the category that includes your household's income before taxes in 2020 . First, was your household income less than $\$ 60,000$ or $\$ 60,000$ or more?

\begin{tabular}{|l|c|}
\hline & Total (\%) \\
\hline Unweighted $n$ & 700 \\
\hline Less than $\$ 60,000.00$ & 48 \\
\hline$\$ 60,000$ or more & 45 \\
\hline Not sure & 1 \\
\hline Refused & 6 \\
\hline
\end{tabular}

a. [IF LESS THAN \$60,000, READ]: so was it..... 
b. [ASK ONLY IF QD10=b, $\$ 60,000$ or more]: so was it.....

\begin{tabular}{|l|c|}
\hline & Total (\%) \\
\hline Unweighted $n$ & 264 \\
\hline$\$ 60,000$ to less than $\$ 75,000$ & 26 \\
\hline$\$ 75,000$ to less than $\$ 100,000$ & 31 \\
\hline$\$ 100,000$ to less than $\$ 125,000$ & 22 \\
\hline$\$ 125,000$ or more & 16 \\
\hline Not sure [DO NOT READ] & 1 \\
\hline Refused [DO NOT READ] & 3 \\
\hline
\end{tabular}

D11. To ensure it is recorded accurately, could you please state your gender?

\begin{tabular}{|l|c|}
\hline & Total (\%) \\
\hline Unweighted $n$ & 700 \\
\hline Male & 49 \\
\hline Female & 51 \\
\hline Other & - \\
\hline
\end{tabular}

For more information about this survey or the methodology, please contact Jennifer Sauer, AARP Research, at jsauer@aarp.org

For more information on this issue in West Virginia, please contact Gaylene Miller, AARP in West Virginia State Director, at gmiller@aarp.org 